\title{
Functioning conditions of the Casale pumping station in Mantova, Italy
}

\author{
C. Capponi ${ }^{1}$, M. Ferrante ${ }^{1}$, M. Pedroni ${ }^{2}$, B. Brunone ${ }^{1}$, S. Meniconi ${ }^{1}$, M. Zaghini ${ }^{2}$, and F. Leoni ${ }^{2}$ \\ ${ }^{1}$ Dipartimento di Ingegneria Civile ed Ambientale, The University of Perugia, via G. Duranti 93, \\ 06125 Perugia, Italy \\ ${ }^{2}$ TeaAcque Srl, Via Taliercio 3, 46100 Mantova, Italy \\ Correspondence to: C. Capponi (caterina.capponi@studenti.unipg.it)
}

Received: 19 December 2013 - Published in Drink. Water Eng. Sci. Discuss.: 10 March 2014

Revised: - - Accepted: 21 October 2014 - Published: 13 November 2014

\begin{abstract}
Pump speed, input power, pressure and discharge data are analyzed for evaluating functioning conditions of Casale pumping station in Mantova, Italy, managed by TeaAcque. A model based on the affinity laws is used to simulate the behavior of the installed variable speed pumps. Quadratic and cubic polynomial curves are used to fit the pump data given by the affinity laws. Such curves allow predicting the efficiency when the functioning conditions change. The relationship between the rotation speed and the efficiency is also derived.
\end{abstract}

\section{Introduction}

Variable speed pumps (VSPs) are widely used in water distribution systems because they can change the functioning conditions more efficiently than fixed speed pumps so as to meet the network requirements. When the system functioning conditions require a change in pump flow and head, the pump speed and characteristic curve vary consequently. In order to simulate the VSP behavior, taking into account the variation of both the system demand and the pump speed, a model based on the affinity laws can be used (Simpson and Marchi, 2013). Such a model allows to simulate the VSP curve by means of three dimensionless quantities, depending on the pump speed. This work is based on the research project involving the University of Perugia and TeaAcque, the manager of the water supply and distribution system in Mantova. Within the project, data acquired at pumping stations are analyzed to improve their efficiency; in this paper attention is focused on the Casale pumping station. The affinity laws are used to model measured data and fit the pump curves. In particular, a quadratic polynomial curve is used to approximate data in terms of dimensionless head and flow, according to Ulanicki et al. (2008). Hydraulic power and efficiency data are fitted by a cubic polynomial curve. Such curves allow predicting the efficiency when the functioning conditions change. The relationship between the rotation speed and the efficiency is also determined. This paper is an extension of the paper by Capponi et al. (2014), presented at the 12th edition of the International Conference on "Computing and Control for the Water Industry - CCWI2013", where a preliminary analysis of the behavior of the Casale pumping station is reported.

\section{Collected data}

The set of data from 22-29 May 2013, provided by TeaAcque, concerns one of the four VSPs installed in the station (Fig. 1): tank level, $h_{\mathrm{L}}$, pressure downstream of the pump, $p_{\mathrm{d}}$, pump flow, $Q$ (measured by means of an electromagnetic flowmeter), and pump speed, $N$, are acquired at $1 / 60 \mathrm{~Hz}$, while the input power, $P_{\text {act }}$, is acquired at $1 / 900 \mathrm{~Hz}$.

With reference to Fig. 2 scheme, where $z_{\mathrm{m}}=2.20 \mathrm{~m}$, since local head losses are negligible, the pump head, $H$, is given by:

$H=\frac{p_{\mathrm{d}}}{\gamma}+z_{\mathrm{m}}-h_{\mathrm{L}}$

with $\gamma=$ water specific weight. To have a general picture of the pumping station functioning conditions, in Fig. 3 the rate of occurrence of $H$ and $Q$ is shown, i.e. the number of data for specific ranges of values. 


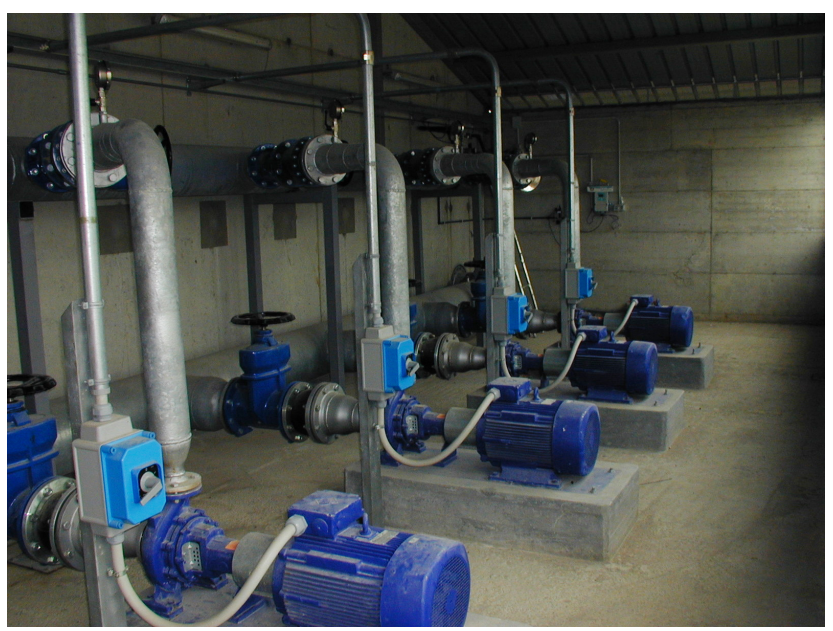

Figure 1. Casale pumping station: the four pumps in parallel.

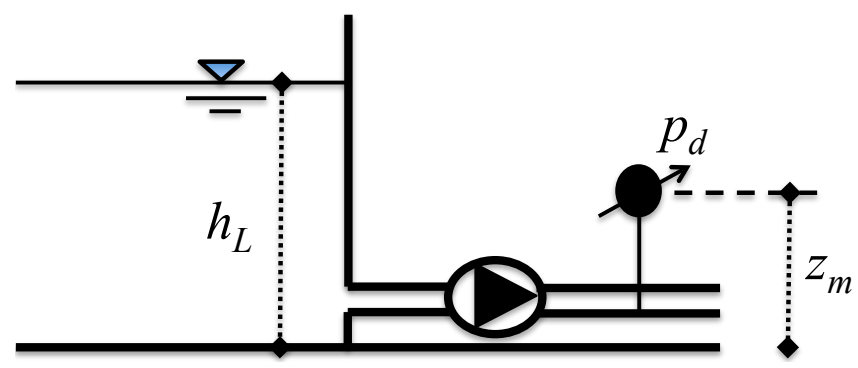

Figure 2. Scheme of the pump setup installation.

Such a histogram points out two zones with the highest rate of occurrence corresponding to pairs of values $(Q, H)$ that represent the most frequent functioning conditions. Such zones merit the attention of managers since any intervention on them gives rise to possible more effective functioning conditions.

In Fig. 4 data are reported in the $Q-H$ plane. Since such data correspond to different pump speeds, they do not lie on a single curve. Hence, to characterize the behavior of the considered pumping station, the rotation speed has to be taken into account.

\section{The model: affinity laws}

The proposed model is based on the following dimensionless quantities defined by the affinity laws:

$$
\begin{aligned}
C_{\mathrm{q}} & =\frac{Q}{N \phi^{3}} \\
C_{\mathrm{h}} & =\frac{g H}{N^{2} \phi^{2}} \\
C_{\mathrm{p}} & =\frac{P}{\rho N^{3} \phi^{5}}
\end{aligned}
$$

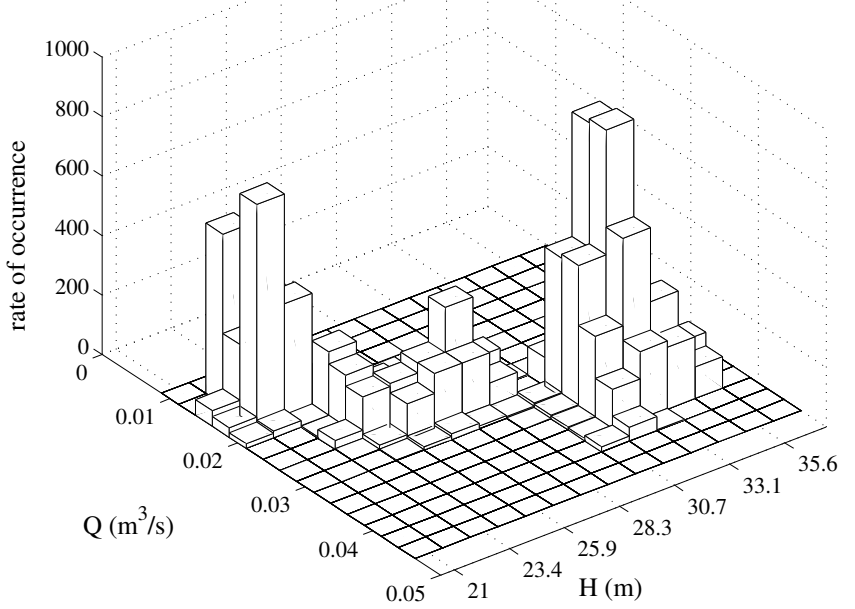

Figure 3. Histogram with the rate of occurrence of $H$ and $Q$.

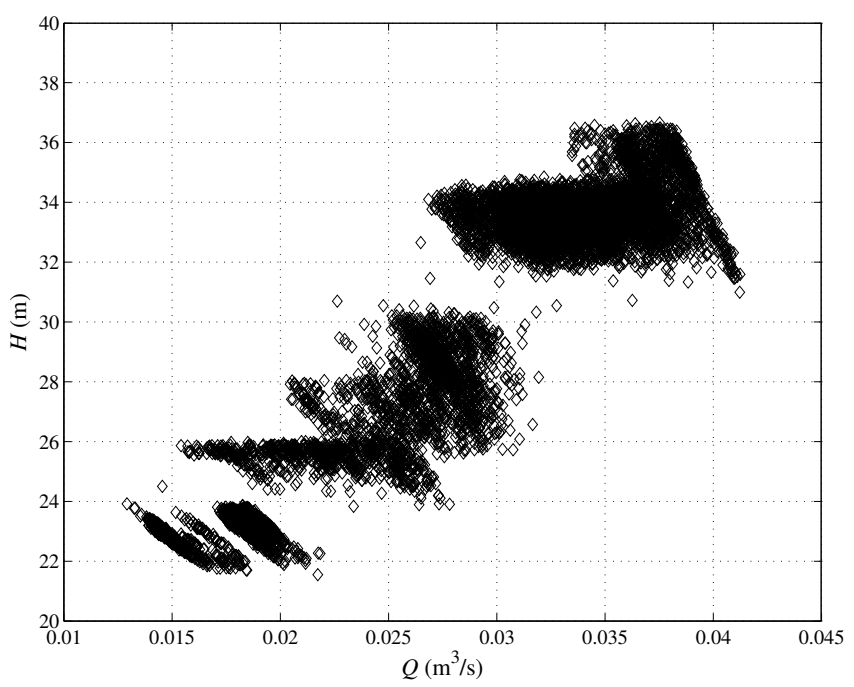

Figure 4. Pump head, $H$, vs. pump flow, $Q$.

where $\rho=$ water density, $g=$ acceleration of gravity, and $\phi=$ impeller diameter. Dimensionless quantities, $C_{\mathrm{q}}, C_{\mathrm{h}}$ and $C_{\mathrm{p}}$ defined by Eqs. (2-4), with $\phi=0.204 \mathrm{~m}$, are more effective in modeling pump curves and in characterizing the behavior of the pumping station, with respect to the dimensional quantities $Q, H$, and $P$ (Simpson and Marchi, 2013). As shown in Fig. 5, by considering $C_{\mathrm{h}}$ and $C_{\mathrm{q}}$, measured data lie on a single curve; specifically the quadratic polynomial proposed by Ulanicki et al. (2008) fits the experimental data with a good agreement $\left(R^{2}=0.9967\right)$ :

$C_{\mathrm{h}}=-485.3915 C_{\mathrm{q}}{ }^{2}+27.2944 C_{\mathrm{q}}+4.8914$

As a useful reference, in Fig. 5 the pump characteristic curve, supplied by the manufacturer, is also plotted, as well as the relevant band of confidence $( \pm 10 \%)$. Differences between 


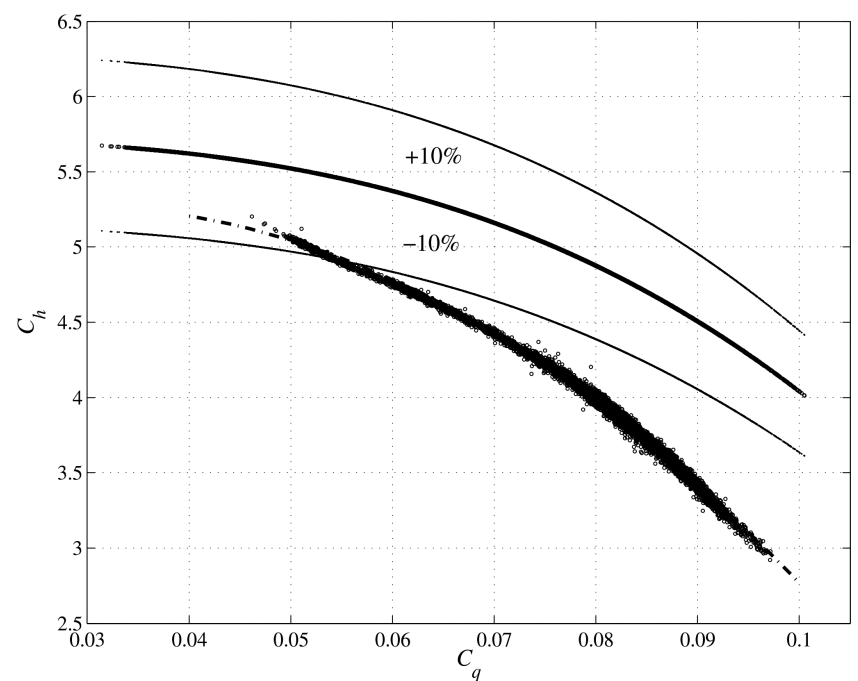

Figure 5. Experimental data, pump characteristic curve (solid line) and the fitting (dashed line) made by Eq. (5) plotted in the $C_{\mathrm{q}}-C_{\mathrm{h}}$ plane (thinner solid lines define the band of confidence given by the manufacturer).

field and manufacturer's data are mainly due to the fact that Eq. (5) curve takes into account more factors related to the system actual functioning conditions.

\section{Efficiency evaluation}

By assuming in Eq. (4) $P=P_{\text {th }}-$ with $P_{\text {th }}=\gamma Q H$ being the theoretical hydraulic power - and $P_{\text {act }}-$ with $P_{\text {act }}=$ measured hydraulic power - the theoretical value, $C_{\mathrm{p}}$, th, and the actual one, $C_{\mathrm{p}}$, act, of $C_{\mathrm{p}}$ can be obtained, respectively. In Fig. 6, $C_{\mathrm{p} \text {, th }}$ and $C_{\mathrm{p} \text {, act }}$ are compared: the $45^{\circ}$ dashed line corresponds to ideal conditions and the distance of the data from it measures the energy dissipation. Precisely from Fig. 6 it can be observed that the $C_{\mathrm{p}}$, act values are about twice the $C_{\mathrm{p}}$, th ones. If $C_{\mathrm{p}}$, th data are plotted against $C_{\mathrm{q}}$ data, the points have a shape that reminds a third degree curve (Fig. 7). In fact, according to Ulanicki et al. (2008), experimental data can be fitted by means of a cubic polynomial curve:

$C_{\mathrm{p}, \mathrm{th}}=-1.124 C_{\mathrm{q}}{ }^{3}+0.1612 C_{\mathrm{q}}{ }^{2}-4.224$

$\times 10^{-3} C_{\mathrm{q}}+2.014 \times 10^{-4}$

Also in this case, the agreement between data and fitting is quite $\operatorname{good}\left(R^{2}=0.9874\right)$.

The efficiency, $\eta$, is a dimensionless parameter that allows evaluating the performance of the pumping station:

$\eta=\frac{P_{\text {th }}}{P_{\text {act }}}$

Values obtained by means of Eq. (7) are reported in the $\eta-C_{\mathrm{q}}$ plane in Fig. 8. Firstly, it can be noted that the $\eta$ values are rarely above 0.6 . It must be said that such an efficiency is a

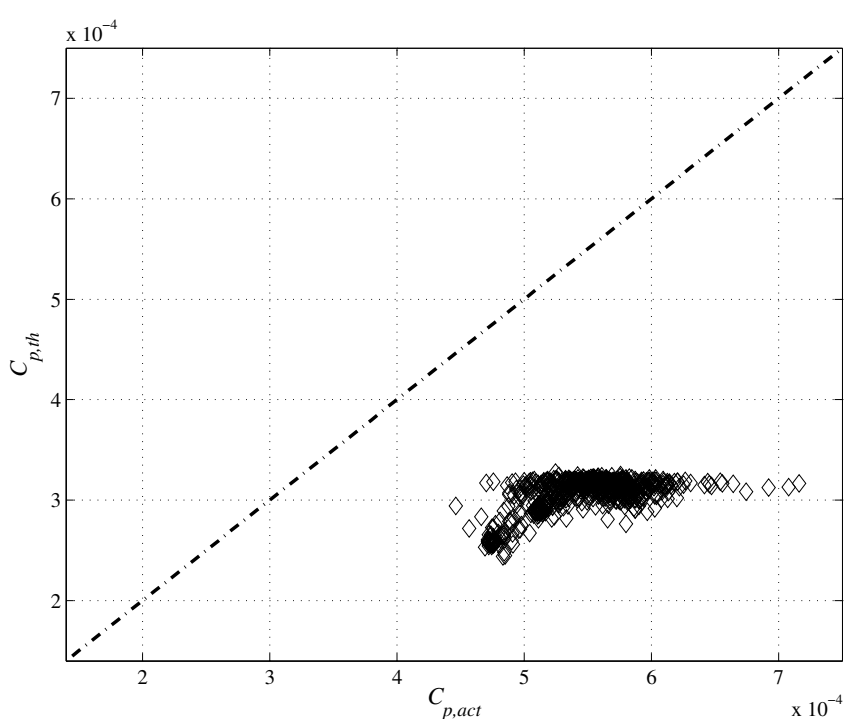

Figure 6. Dimensionless streamflow power, $C_{\mathrm{p}}$, th vs. dimensionless input power, $C_{\mathrm{p}}$, act. The dashed line is the $45^{\circ}$ line.

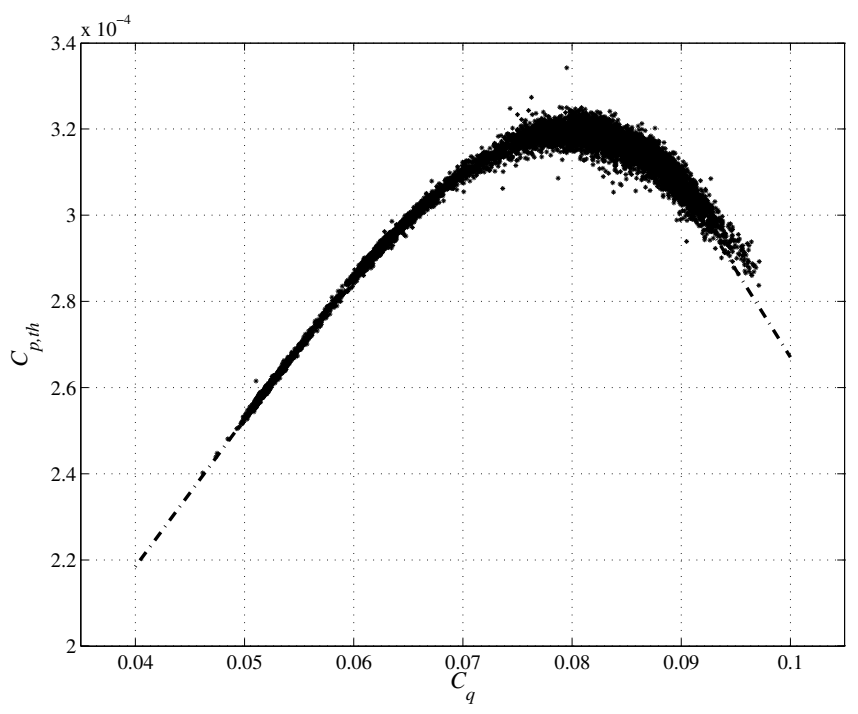

Figure 7. Dimensionless streamflow power, $C_{\mathrm{p}}$, th, vs. dimensionless flow, $C_{\mathrm{q}}$ : experimental data and fitting.

"wire to water" efficiency. In fact it takes into account many factors and then such quite low values are not unexpected. Secondly, although data spreading is higher than in the previous graphs, a fitting can be made by a cubic polynomial curve:

$\eta=-903.3 C_{\mathrm{q}}{ }^{3}+87.44 C_{\mathrm{q}}{ }^{2}+1.625 C_{\mathrm{q}}+0.3437$

with $R^{2}=0.772$. For the sake of clarity, both fittings of $C_{\mathrm{h}}$ (Fig. 5) and $\eta$ (Fig. 8) in terms of $C_{\mathrm{q}}$ are plotted in Fig. 9. These two curves allow to characterize the Casale pumping station in its actual functioning conditions. 


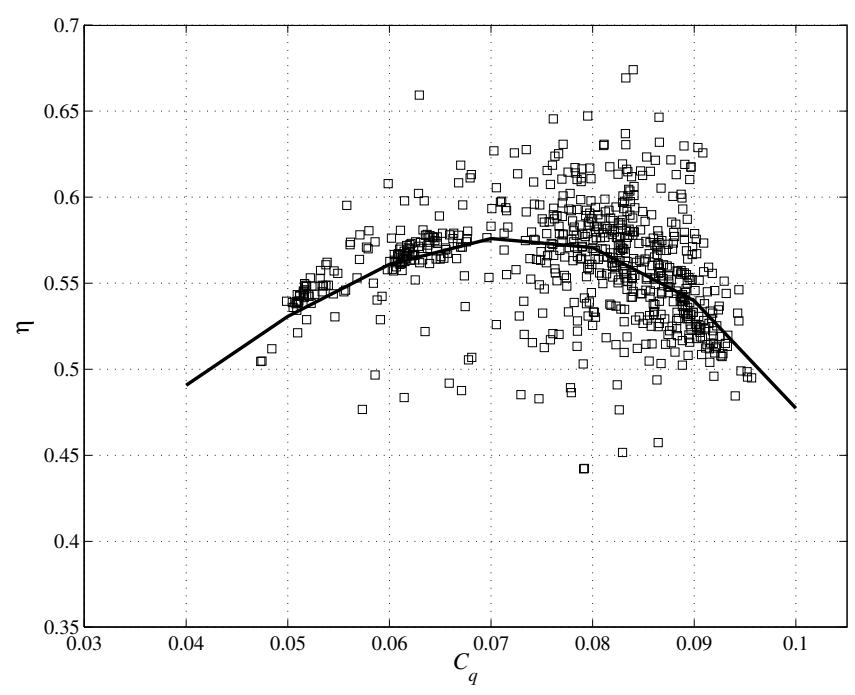

Figure 8. Efficiency values in terms of dimensionless flow, $C_{\mathrm{q}}$ : experimental data and fitting.

When the system functioning conditions require a change in pump flow and head, the pump speed and characteristic curve are varied consequently by means of an inverter drive - also referred to as "variable frequency drive" - installed in the system. In particular, the pump speed is adjusted so that the pump works at a chosen pump head value within a certain time slot: this value is usually called "set point". So, for each hour of the day, a set point is determined. Within each interval defined by the set points, the pump speed, $N$, increases with the flow, according to the trend shown in Fig. 10. On this figure also the efficiency values against flow are plotted. It can be observed that for both the lowest and highest values of $N$ and $Q, \eta$ values decrease. By means of this analysis it is possible to predict the behavior of the pumping station in terms of efficiency, for the given set points.

\section{Conclusions}

Measurements of pump speed and input power, besides pressure and flow data, can be useful for management purposes. Pump speed data allow to scale hydraulic pump characteristics, i.e. pump head and flow, and to model the pump behavior, in terms of pump characteristic curves, according to affinity laws. In this paper a set of measurements executed at the Casale pumping station is considered, for the duration of a week. Experimental data are firstly analyzed in terms of pump head and flow, evaluating the rate of occurrence, in order to find the main functioning conditions of the pumping station. Secondly, data are analyzed using the three dimensionless quantities introduced by affinity laws. Data in the dimensionless plane $C_{\mathrm{q}}-C_{\mathrm{h}}$ can be fitted with a quadratic polynomial with a quite good agreement. The dimensionless streamflow power, $C_{\mathrm{p}}$, th, can be expressed in terms of $C_{\mathrm{q}}$ by a cubic polynomial, with a quite good agreement. The

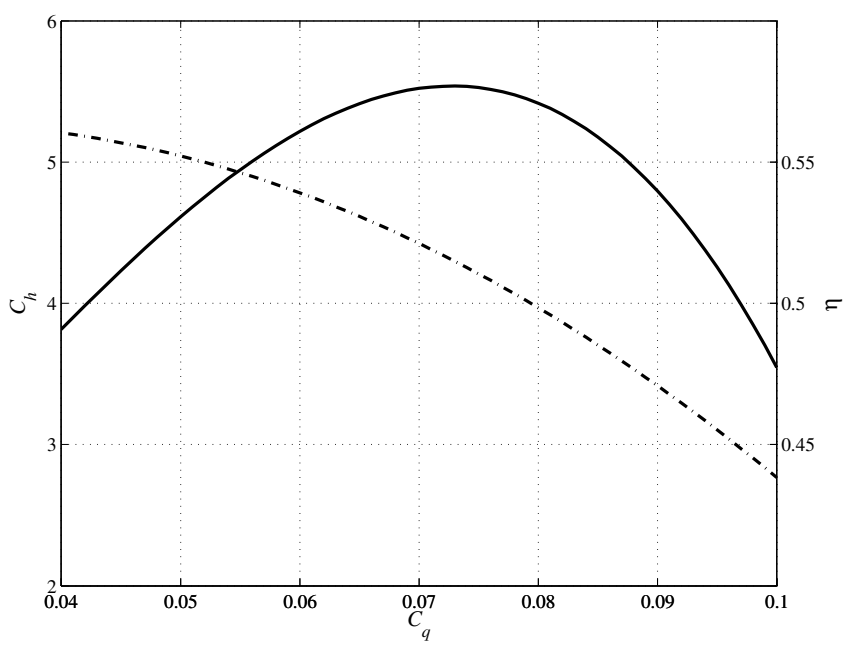

Figure 9. Fittings of data of Figs. 5 (dashed line) and 8 (continuous line) plotted on the same plane.

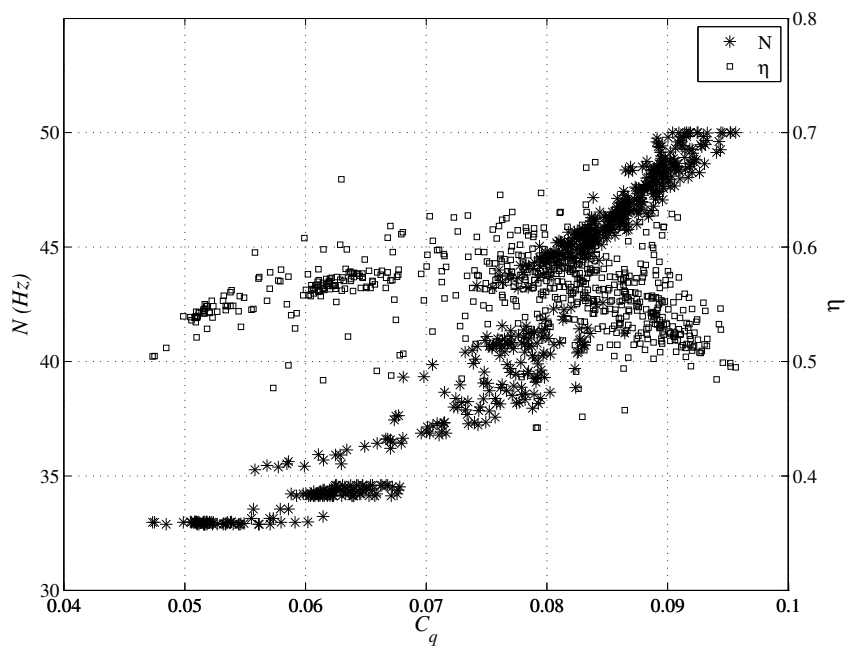

Figure 10. Pump speed, $N$, and efficiency, $\eta$, values against dimensionless flow, $C_{\mathrm{q}}$.

streamflow power is also compared with the measured input power: the ratio between these two quantities allows evaluating the "wire to water" efficiency, $\eta$, of the pumping station. The $\eta$ experimental data show a high spreading and the fitting is characterized by a worse agreement if compared with the other fittings. Trends of pump speed and efficiency against pump flow, if plotted on the same figure, show how the efficiency changes with the variation in the rotation speed, which is related to the setting of the inverter drive. Combining this analysis with a larger set of data will allow to improve the pumping station functioning conditions and then to reduce the costs of the system management. The knowledge of the network behavior could be combined with the results shown in this paper in order to predict the response of the network to any change in the pumping station functioning conditions. 
Acknowledgements. This research has been supported by the Italian Ministry of Education, University and Research (MIUR) under the Projects of Relevant National Interest "Advanced analysis tools for the management of water losses in urban aqueducts", "Tools and procedures for an advanced and sustainable management of water distribution systems" and Fondazione Cassa Risparmio Perugia under the project "Hydraulic characterization of innovative pipe materials (no. 2013.0050.021)".

Edited by: A. Grefte

\section{References}

Capponi, C., Ferrante, M., Pedroni, M., Brunone, B., Meniconi, S., Zaghini, M., and Leoni, F.: Real data analysis and efficiency of the TEA Mantova Casale (Italy) variable-speed pumping station, 12th Int. Conference on "Computing and Control for the Water Industry - CCWI2013”, Perugia, Procedia Engineering, Elsevier, 70, 248-255, 2014.

Simpson, A. R. and Marchi, A.: Evaluating the approximation of the affinity laws and improving the efficiency estimate for variable speed pumps, J. Hydraul. Engin., 139, 1314-1317, 2013.

Ulanicki, B., Kahler, J., and Coulbeck, B.: Modeling the efficiency and power characteristics of a pump group, J. Water Resour. Plann. Manage., 134, 88-93, 2008. 